Prix Servant 2011 de l'Académie des sciences

\title{
Superconducting quantum point contacts
}

\section{Contacts ponctuels quantiques supraconducteurs}

\author{
L. Bretheau, Ç. Girit, L. Tosi, M. Goffman, P. Joyez, H. Pothier, D. Esteve, C. Urbina*,1 \\ Quantronics Group, Service de Physique de l'État Condensé (CNRS, URA 2464), IRAMIS, CEA-Saclay, 91191 Gif-sur-Yvette cedex, France
}

\section{A R T I C L E I N F O}

\section{Article history:}

Received 6 September 2011

Accepted after revision 2 December 2011

Available online 23 December 2011

\section{Keywords:}

Atomic contacts

Superconductivity

Quantum transport

\section{Mots-clés :}

Contacts atomiques

Supraconductivité

Transport quantique

\begin{abstract}
A B S T R A C T
We review our experiments on the electronic transport properties of atomic contacts between metallic electrodes, in particular superconducting ones. Despite ignorance of the exact atomic configuration, these ultimate quantum point contacts can be manipulated and well characterized in-situ. They allow performing fundamental tests of the scattering theory of quantum transport. In particular, we discuss the case of the Josephson effect.

(C) 2011 Académie des sciences. Published by Elsevier Masson SAS. All rights reserved.

R É S U M É

Nous décrivons un ensemble d'expériences de transport électronique que notre groupe a réalisées sur des contacts atomiques entre des électrodes métalliques, en particulier dans l'état supraconducteur. Bien que leur configuration atomique exacte ne soit pas connue, ces contacts ponctuels quantiques peuvent être manipulés et caractérisés in-situ, et permettent de mener à bien des tests fondamentaux de la théorie en diffusion du transport quantique. Nous discutons en particulier le cas de l'effet Josephson.
\end{abstract}

(c) 2011 Académie des sciences. Published by Elsevier Masson SAS. All rights reserved.

\section{Introduction}

For significant quantum effects to appear in the transport properties of a conductor, they must be shorter than the distance over which an electron propagates without losing its quantum coherence, which for usual metals at low temperatures $(<1 \mathrm{~K})$ is on the order of microns. The exploration of this quantum transport regime started in the early 1980s with the observation of Aharonov-Bohm interferences in submicron metallic rings [1]. A very powerful point of view, put forward by R. Landauer [2,3], describes a transport experiment across a coherent conductor as the scattering of the quantum electronic waves injected by the contact probes. In other words, the conductor is viewed as an electronic waveguide, whose modes, called "conduction channels", are each characterized by a transmission probability. For example, the total conductance is given by the famous Landauer formula: $G=G_{0} \sum_{1}^{N} \tau_{i}$, where $N$ is the number of channels, the $\tau_{i}$ are the individual transmission probabilities, and $G_{0}=2 e^{2} / h \sim 77 \mu \mathrm{S}$ the conductance quantum. In fact, in systems with non-interacting quasiparticles all transport quantities can be expressed in terms of the transmission probability set $\left\{\tau_{i}\right\}$, which is viewed in this framework as the "Personal Identity Number" (PIN code) of the conductor [4]. For most systems, the number of conduction channels is very large, and the description of transport can only be based on a distribution function for the transmission eigenvalues. However, the number of channels can be significantly reduced if the lateral dimensions of a conductor become

\footnotetext{
* Corresponding author.

E-mail address: cristian.urbina@cea.fr (C. Urbina).

1 The Prix Servant 2011 has been awarded to Cristian Urbina by the French Académie des sciences.
} 
comparable to the Fermi wavelength of the electrons. This was demonstrated in 1988 using constrictions tailored in the two-dimensional electron gas of a semiconductor heterostructure, with a width adjustable on the scale of the Fermi wavelength $(\sim 50 \mathrm{~nm})$ by means of an electrostatic gate $[5,6]$. In these "quantum point contacts", as the constriction width is continuously decreased, a descending staircase conductance curve is observed, beautifully illustrating the closing of the channels one after another. Since then much activity has been devoted worldwide to the investigation of transport through a broad range of coherent conductors (diffusive and ballistic conductors, quantum dots, nanowires, individual molecules like carbon nanotubes) connected to reservoirs in the normal or in the superconducting state [4].

Among the various systems investigated, atomic-size contacts [7] between three-dimensional metallic electrodes play an important role. These contacts have been accessible since the early years of STM [8], and more stable ones were achieved at the beginning of the 1990s by the group of Jan van Ruitenbeek at Leiden using the break-junction technique [9,10]. Since their characteristic transverse dimensions are of the order of the Fermi wavelength (typically $0.2 \mathrm{~nm}$ ), atomic contacts accommodate only a small number of channels and behave as quantum point contacts even at room temperature. An interesting difference with respect to quantum point contacts in 2D electron systems is that superconductivity can be built-in with the proper choice of material. The discovery by our group in 1997 that the PIN code could be accurately measured for every contact [11] paved the way to a new generation of quantum transport experiments in which the measured transport quantities could be compared to the theoretical predictions with no adjustable parameters. As an example, we describe here our experiments on the supercurrent flowing through atomic-size contacts. They probe the main concepts of the "mesoscopic" theory of the Josephson effect, i.e. the theory in terms of the transmission probability of the channels, that we sketch in Section 3. Before discussing previous and on-going experiments in Sections 4 and 5 respectively, we describe the microfabrication technique that has made possible the experiments and the procedure we use to determine the PIN code of atomic-size contacts in Section 2.

\section{Production and characterization of atomic contacts}

\subsection{Microfabricated break junctions}

In order to produce atomic contacts we developed the microfabricated break-junction (MBJ) technique [12]. Using electron beam lithography and reactive ion etching, a metallic bridge clamped to an elastic substrate is suspended over a few microns between two anchors (see top-left panel of Fig. 1). The bridge has a constriction at the center with a diameter of approximately $100 \mathrm{~nm}$. Using the mechanism shown in the top-right panel of Fig. 1 the substrate is first bent until the bridge breaks at the constriction. This is performed under cryogenic vacuum so that there is no contamination of the two resulting electrodes which are then gently brought back into contact. Afterward, the contact can be repeatedly made and broken at will.

The bottom panel of Fig. 1 displays a typical conductance evolution observed while opening an aluminum MBJ. The conductance evolves through a series of slanted plateaus and sharp steps. The conductance on the last plateau before full opening and the height of the previous steps is of the order of the conductance quantum $G_{0}$. Although similar staircase patterns are observed every time the experiment is performed, the horizontal extension (of the order of a fraction of nanometer) and the vertical position of the plateaus are not always the same for subsequent compression-extension cycles. These general features have been observed by many groups for a variety of metals under different experimental conditions (temperature, technique used to produce atomic size contacts, rate of compression and extension). The typical conductance on the last plateau, the typical lengths of the plateaus, and the behavior within the plateaus are characteristic of each material [7].

The succession of plateaus and conductance jumps is directly related to the dynamics of the atomic configuration of the contact. Combined STM-AFM experiments that measure the force between the tip and the surface simultaneously with the conductance have directly shown that on a plateau the atomic configuration is only elastically deformed while a conductance jump results from an abrupt reconfiguration of the atoms at the contact accompanied by stress relief [13,14]. Molecular dynamics simulations [15-17] confirm this interpretation of the staircase pattern. These were the first clues that the smallest contacts are indeed made of a single atom. In the case of gold, one-atom contacts and atomic chains [18] have even been observed directly with high resolution Transmission Electron Microscopes [19-21].

As compared to other techniques, microfabricated break junctions present several major advantages essential to the realization of the experiments presented here. First, atomic-size contacts fabricated this way are extremely stable and can be maintained in the same configuration for weeks. Second, with a given suspended bridge many different contacts can be created in-situ, allowing exploration of the physics of interest for a broad range of transmission coefficients. Finally, the flexibility offered by microfabrication techniques makes possible embedding the contacts into on-chip circuits.

\subsection{Determination of the PIN code}

Our first major result was devising a method to determine not only the number of channels accommodated by a contact but also its PIN code with good accuracy [11,22]. This was achieved by measuring the current-voltage characteristic $I(V)$ 

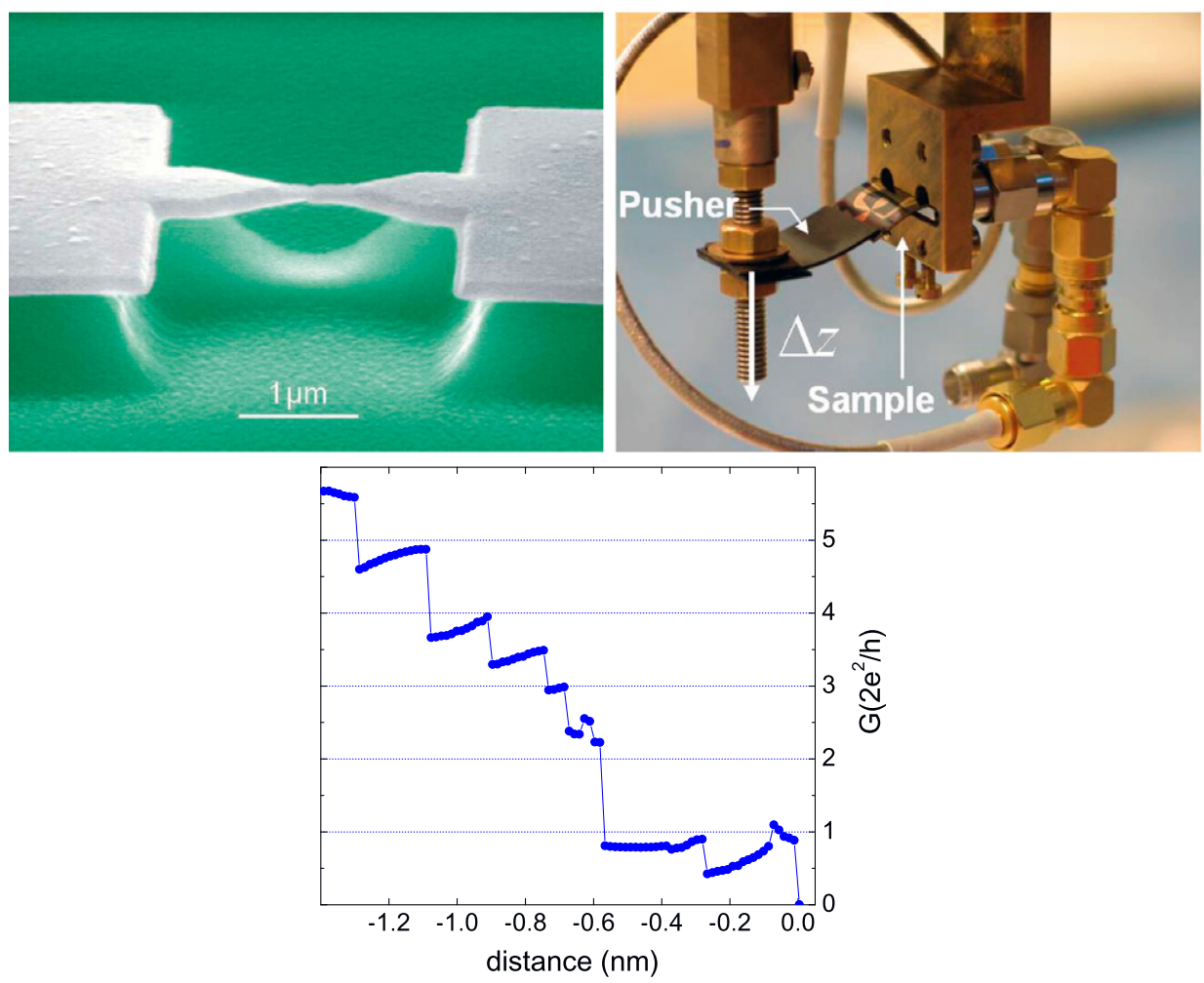

Fig. 1. Principle of the microfabricated break-junction technique. Top left: A thin microfabricated aluminum bridge is broken by bending the substrate on which it is fabricated. Top right: Example of a bending mechanism allowing microwave measurements. The ensemble is placed in cryogenic vacuum and cooled below $100 \mathrm{mK}$. A rod, driven by a room temperature dc motor, pushes the free end of the sample which is firmly clamped on the opposite side against two microwave SMA launchers. Typically, a vertical displacement $\Delta z=1 \mu \mathrm{m}$ of the pushing rod results in a 10-100 pm change of the distance between the electrodes of the bridge. Bottom: Conductance of a bridge, in units of the conductance quantum, as a function of displacement when pulling the electrodes apart. The origin of the displacement was set arbitrarily at the point where the bridge actually breaks and the conductance starts decreasing exponentially signaling the tunnel regime. Measurements were performed at $50 \mathrm{mK}$ under a magnetic field of $200 \mathrm{mT}$ to suppress superconductivity in the aluminum films.

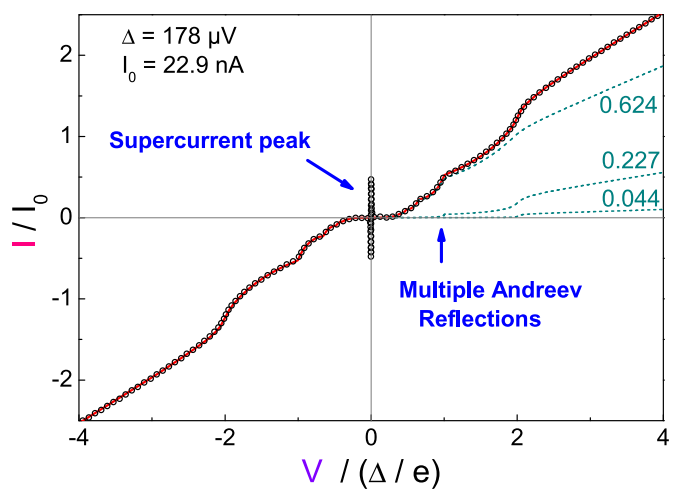

Fig. 2. $I(V)$ characteristics of an atomic contact. Open symbols: experimental data. Continuous line: best fit resulting from the sum of three single channel characteristics (dashed lines) calculated using the theory of MAR and the transmission values indicated on the right edge.

of the contact in the superconducting state (see Fig. 2). ${ }^{2}$ At exactly zero voltage a finite current, i.e. a supercurrent, can flow. Above some bias current, the system switches to a finite voltage state, i.e. the current becomes dissipative. The voltage and transmission dependence of the dissipative current through a weak link was explained for the first time in the early 1980s in terms of Multiple Andreev Reflections (MAR) [23,24]. A full quantum theory of MAR for a single channel of

\footnotetext{
${ }^{2}$ Current-noise measurements in the normal state can also be used to determine the PIN code, but only for contacts containing no more than two channels [7].
} 
arbitrary transmission was achieved in the mid 90s [25-27]. Three numerically calculated current-voltage characteristics resulting from these MAR processes are shown in Fig. 2. These elementary curves are highly non-linear below twice the superconducting gap $\Delta$ and present current steps at voltages $2 \Delta / e n$, with $n$ an integer, which mark the onset of MAR of different orders. The $n$-th order process involves the transfer of $n$ electrons, and in a given channel its intensity varies as the $n$-th power of the transmission. Consequently, the $I(V)$ characteristic depends on all powers of every transmission coefficient and carries sufficient information to reconstruct the PIN code. The determination of the PIN code of any atomicsize contact is achieved by decomposing the measured total current into a series of such elementary characteristics, each of them corresponding to a well defined transmission probability. The individual transmission probabilities of the channels are adjusted so as to get the best fit of the measured current-voltage characteristic [28]. This automatically yields the number of channels having a non-negligible transmission. The uncertainty on the transmissions obtained by this fitting procedure depends on the uncertainty in the measurement of both voltage and current, on the transmission of the channels participating in the contact, and on the environmental impedance. Typically, for channels with a large transmission the relative uncertainty achieved on $\tau_{i}$ is of the order of $0.1 \%{ }^{3}$ For channels having low transmissions $(\tau<0.05)$, the procedure fails to disentangle the contributions of the different channels, and yields a larger error bar (see Chapter 1 of [22] for details).

Using this method we showed that for one-atom contacts the number of conduction channels is directly related to the number of valence orbitals of the metal [29]. For aluminum and lead, which have $p$-electrons at the Fermi level, three channels were found to contribute to the conductance of the smallest contacts. For Niobium, a transition metal with $s$ - and $d$-electrons, five conduction channels were found. In the case of gold, with one $s$-electron, and for which superconductivity was induced through the proximity effect by intimate contact with a thick aluminum layer, we found that only one conduction channel contributes to the conductance on the lowest conductance plateau [30].

\section{Mesoscopic theory of the Josephson effect}

In 1962, Josephson predicted that a dc current could flow at zero bias voltage between two superconducting electrodes coupled by a tunnel barrier [31]. This supercurrent results from the coherent transfer of Cooper pairs and is driven by the phase difference $\delta=\delta_{L}-\delta_{R}$ between the superconducting order parameters of the two electrodes. The phase difference is related to the voltage difference $V$ between the electrodes by the Josephson relation $\dot{\delta}=2 \pi V / \phi_{0}$, with $\phi_{0}=h / 2 e$ the flux quantum. ${ }^{4}$ Since its prediction, the Josephson current has been observed in a great variety of systems involving superconducting electrodes connected by a "weak link". A weak link can be an insulating layer, as originally proposed by Josephson, or any quantum coherent conductor, such as a short normal (diffusive or ballistic) metallic wire, a point contact, a carbon nanotube, or a graphene layer [32]. A great deal of theoretical activity has been devoted to relate the maximum (or critical) supercurrent $I_{0}$ of a weak link to its normal state properties. A unifying theoretical description providing this relation for an arbitrary structure in terms of its transmission PIN emerged in the 1990s and is based on the concept of Andreev bound states $[33,34]$.

Due to the existence of a gap in the quasiparticle density of states in a superconductor, an electron impinging from a normal metal into a superconducting electrode with an energy $|E|<\Delta$ cannot enter it as such, and should in principle be reflected back. However, if there is no barrier at the interface it will be "Andreev reflected" as a hole at the same energy and moving in the opposite direction, adding a Cooper pair to the superconductor [35].

\subsection{Andreev bound states}

The formation of Andreev bound states is illustrated for a single channel in Fig. 3 [36].

In a perfectly transmitting channel, a right-moving electron is Andreev reflected at the interface with the right superconducting electrode into a left-moving hole, and acquires an energy dependent phase shift $\arccos (E / \Delta)+\delta_{R}$ where $\delta_{R}$ is the phase of the local superconducting order parameter. In turn, this left-moving hole is Andreev reflected as a right-moving electron at the left electrode, removing a Cooper pair from it. These successive reflections interfere constructively, like in a Fabry-Pérot interferometer, when the global phase shift acquired along one round-trip is an integer multiple of $2 \pi$. Of course, a similar process occurs for left-moving electrons reflected as right-moving holes, and eventually two resonant quasiparticle states $|\leftarrow\rangle$ and $|\rightarrow\rangle$ appear in the channel region. Their phase-dependent energies lie within the superconducting energy gap and are symmetric with respect to the Fermi level.

If the channel is not perfectly transmitting $(\tau<1)$, a right-moving electron can also be simply reflected as a left-moving electron. The existence of a finite reflection probability thus couples the two reflectionless states. The two resulting states, denoted $\{|-\rangle,|+\rangle\}$, are called the "Andreev states", and have energies $\mp E_{A}$ shown in Fig. 4, where:

$$
E_{A}(\delta, \tau)=\Delta \sqrt{1-\tau \sin ^{2}\left(\frac{\delta}{2}\right)}
$$

\footnotetext{
${ }^{3}$ For sufficiently low environment impedance to avoid significant Coulomb blockade effects.

${ }^{4}$ From the circuit point of view, these properties make the Josephson element to behave as a non-linear inductor. Together with the junction intrinsic capacitance it thus form an anharmonic resonator. The oscillation mode is known as the "plasma resonance".
} 


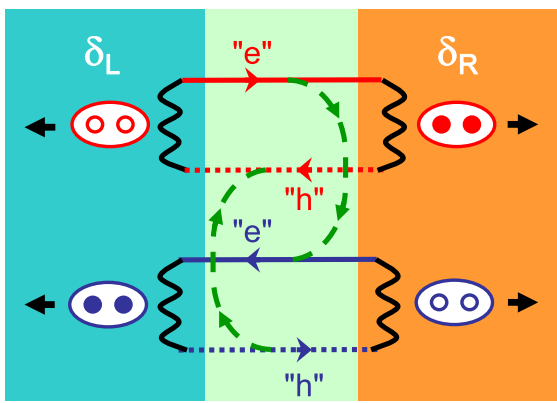

Fig. 3. Schematic representation of the microscopic processes leading to the formation of two Andreev bound states in a short channel connecting two superconducting electrodes with different phases $\delta_{L}$ and $\delta_{R}$. The wiggly lines represent an Andreev reflection in which an electron (hole) is reflected as a hole (electron) acquiring the local superconducting phase. The upper (lower) loop corresponds to the transfer of Cooper pairs to the right (left). When the transmission probability of the channel is below unity, in addition to the Andreev reflection processes, normal reflection processes (dashed lines) connects electron (hole) states traveling in opposite directions.
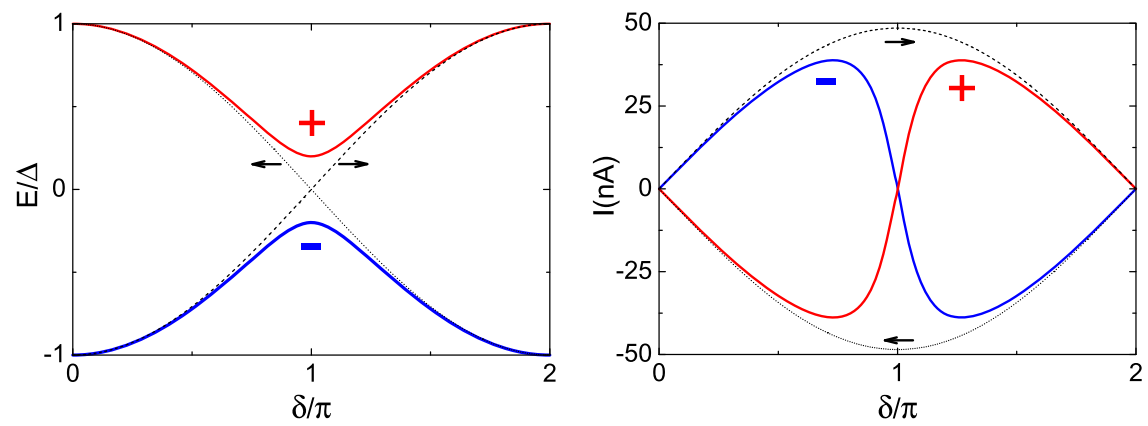

Fig. 4. Left: Energy of Andreev states $|-\rangle$ and $|+\rangle$ in a single channel of transmission $\tau=0.96$ (solid curves) as a function of the superconducting phase difference $\delta$ across it. The dashed curves represent those in a reflectionless channel $|\leftarrow\rangle$ and $|\rightarrow\rangle$. Right: Supercurrent carried by the upper $|+\rangle$ and lower $|-\rangle$ Andreev states calculated for $\Delta=200 \mu \mathrm{eV}$ (full lines). The dashed curves are the currents for the reflectionless channel.

Let us point out that in the tunnel limit $\tau \rightarrow 0$, this expression correctly yields the $\cos (\delta)$ dependence of the coupling energy predicted by Josephson for a tunnel junction. The transition frequency between the two Andreev states reaches its minimum $(2 \Delta / h) \sqrt{1-\tau}$ at $\delta=\pi$.

Because the number of transferred pairs and the phase difference are conjugated variables, these states carry supercurrents given by:

$$
I_{ \pm}(\delta, \tau)=\frac{2 \pi}{\phi_{0}} \frac{\partial E_{ \pm}(\delta)}{\partial \delta}=\mp \frac{e \Delta}{2 \hbar} \frac{\tau \sin \delta}{\sqrt{1-\tau \sin ^{2}\left(\frac{\delta}{2}\right)}}
$$

and shown in the right panel of Fig. 4. Since at a given phase the two Andreev bound states carry the same current but in opposite directions, the net supercurrent results from the imbalance of their populations. At zero temperature, only the lower energy state is occupied and the maximum (or critical) supercurrent is given by

$$
I_{0}(\tau)=\frac{e \Delta}{\hbar}(1-\sqrt{1-\tau})
$$

which is not simply proportional to $\tau$ and thus neither to the normal state conductance. In aluminum, where the superconducting gap $\Delta$ is typically of the order of $200 \mu \mathrm{eV}$, this current reaches at most $\simeq 50 \mathrm{nA}$ for a single perfectly transmitting channel, and the transition frequency is in the microwave range.

\subsection{Excitation spectrum}

It is important to note that the pair of Andreev bound states leads locally to an excitation spectrum (Fig. 5) containing, at an energy $E_{A} \leqslant \Delta$ above the ground state, a discrete spin-degenerate doublet [37]. There are four possible occupation configurations for the system. In the global ground state configuration, the total energy contains a phase dependent term $-E_{A}(\delta, \tau)$ arising from the lowest lying localized Andreev state, which is occupied by a spin-singlet pair of electrons and gives rise to a supercurrent $I_{-}=-\varphi_{0}^{-1} \partial E_{A} / \partial \delta$, with $\varphi_{0}=\hbar / 2 e$. Then, there are two "odd" configurations (spin $1 / 2$ ) with a single excitation of the doublet at $E_{A}$, corresponding to a quasiparticle trapped in the one-channel constriction. In this case the global energy is zero, phase independent, and the total supercurrent is zero. Finally, there is another spin-singlet 


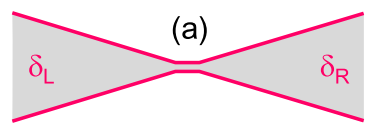

(b)

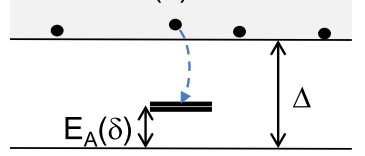

(c)

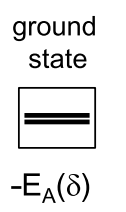

1-qp excitations

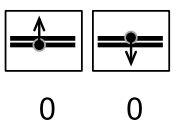

excited singlet

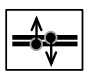

$\mathrm{E}_{\mathrm{A}}(\delta)$ (d)

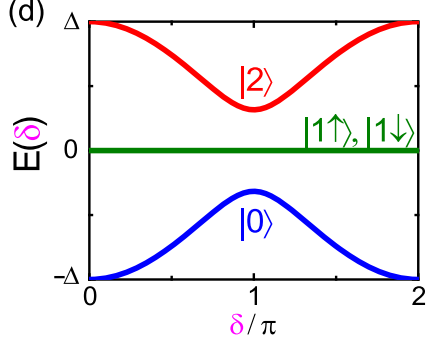

Fig. 5. (a) Short one-channel constriction between two superconducting electrodes (phase difference $\delta=\delta_{L}-\delta_{R}$ ). (b) Excitation spectrum: besides the usual continuum of excited states above the energy gap $\Delta$, that extends all across the structure, there is at the constriction a discrete Andreev spin-degenerate doublet with energy $E_{A}(\delta)$ above the ground state, where quasiparticles can get trapped. (c) Four possible configurations of the Andreev doublet at the constriction: in the global ground configuration, labeled $|0\rangle$, the doublet is empty (it corresponds to just the ground Andreev state $|-\rangle$ of Fig. 4 being occupied); in the two odd configurations, each with definite spin $\pm 1 / 2$ and labeled $|1 \uparrow\rangle$ and $|1 \downarrow\rangle$, one quasiparticle is added to the contact (they correspond to the Andreev states of Fig. 4 being both occupied or both empty) [37]; the last configuration is a spin-singlet double excitation, labeled $|2\rangle$ (it corresponds to just the excited Andreev state $|+\rangle$ of Fig. 4 being occupied). (d) Total energy of the four configurations vs phase. The energy of the ground configuration $|0\rangle$ is that of the lowest Andreev level $-E_{A}$. The two odd configurations, $|1 \uparrow\rangle$ and $|1 \downarrow\rangle$, have zero energy. The double excitation configuration $|2\rangle$, has the energy of the excited Andreev state $+E_{A}$.

configuration with a double excitation which carries a supercurrent $I_{+}=+\varphi_{0}^{-1} \partial E_{A} / \partial \delta$ exactly opposite to the one in the ground configuration. Hence the supercurrent through the constriction is a probe of the excitation configuration of the system.

\subsection{Voltage bias and Andreev states}

When the structure is voltage-biased at $V \ll \Delta / e$, the phase varies linearly with time at a speed $\dot{\delta}=2 \pi V / \phi_{0}$. When the latter is low enough, one can assume that the Andreev levels move adiabatically within the superconducting gap $\Delta$. As the motion is periodic, there is no energy transfer to the system on average and a purely ac current flows. This corresponds to the second striking prediction of Josephson, the ac Josephson effect. For larger voltages, and therefore larger speeds, nonadiabatic transitions (Landau-Zener type) arise between the Andreev levels, giving rise to the onset of the dissipative MAR current discussed in Section 2.2 [26]. The onset of this dissipative current depends on the channel transmission.

\section{Probing the Josephson effect in atomic contacts}

For an arbitrary quantum coherent conductor the phase-driven supercurrent is governed by the occupation numbers of the Andreev states in all channels. At very low temperature, when only the lower energy Andreev state of each channel is occupied, the current-phase relation is:

$$
I\left(\delta,\left\{\tau_{i}\right\}\right)=\frac{e \Delta}{2 \hbar} \sum_{i=1}^{N} \frac{\tau_{i} \sin (\delta)}{\sqrt{1-\tau_{i} \sin ^{2}\left(\frac{\delta}{2}\right)}}
$$

This is one of the fundamental predictions of the mesoscopic theory of the Josephson effect that we have probed in our experiments with atomic contacts.

In a first series of experiments carried out on current-biased contacts [38], we measured the average supercurrent $I_{S}$ at which the system switches to the dissipative branch. In this case, the phase is not an externally tunable parameter, but a dynamical variable moving in a potential landscape fixed by the total Josephson coupling energy and submitted to random fluctuations imposed by the biasing circuit. As a consequence the switching current is always smaller than the critical current $I_{0}$. However, because we embedded the contact in a properly designed on-chip circuit, dissipation and thus fluctuations were under control. Most of the experimental results could be well understood by considering just the contribution of the ground Andreev state of each channel, but evidence for non-adiabatic transitions between Andreev states was observed for nearly ballistic contacts.

However, these experiments were actually only an indirect test of the prediction of Eq. (3), since the phase was not an external parameter that could be swept over its entire range. A thorough test of the theory actually requires measuring, for a given atomic contact, both its current-phase relation and its $I(V)$ characteristic (to determine its PIN code). These two measurements require contradictory biasing conditions. For the former, the atomic contact must be phase-biased, which requires inserting it in a small superconducting loop threaded by a magnetic flux. For the latter, one needs to voltage-bias the same atomic contact, which cannot be achieved if it is shunted by the superconducting loop. Obviously, one needs a superconducting reversible switch in order to toggle between the two biasing conditions without disturbing the atomic contact. 

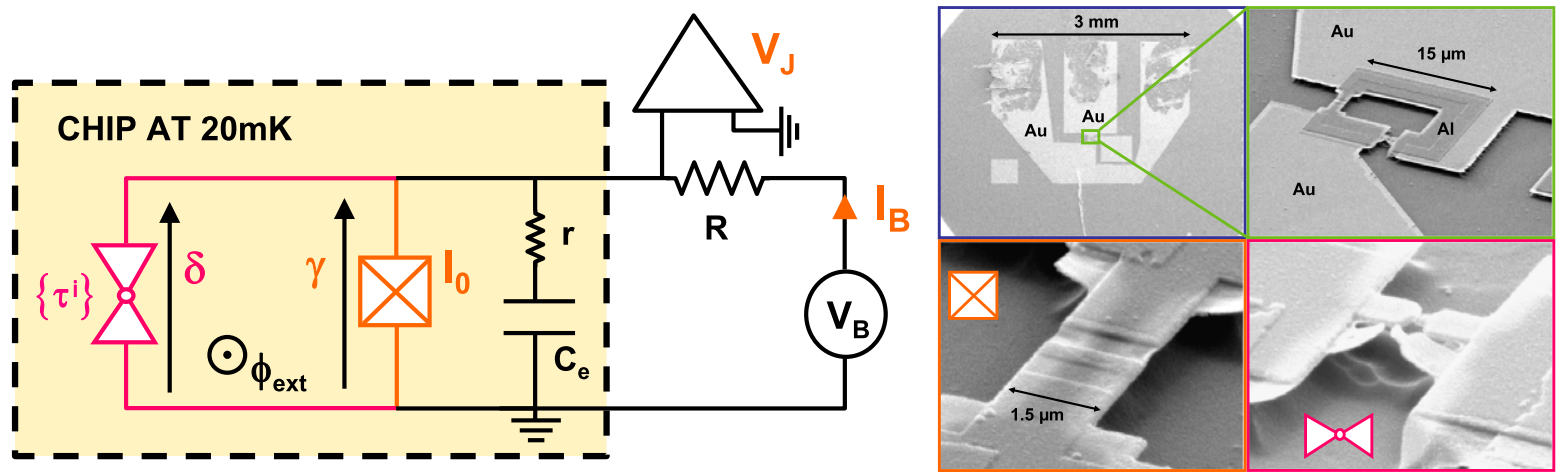

Fig. 6. Left: An aluminum atomic contact in parallel with a Josephson junction having a large critical current $I_{0}=310 \mathrm{nA}$ forms an atomic-SQUID. An on-chip capacitor $C_{e} \simeq 20 \mathrm{pF}$ lowers the plasma frequency of the junction to $1.1 \mathrm{GHz}$ and the associated resistance $r \sim 0.5 \Omega$ damps the dynamics of the phase. The SQUID is biased through a resistor $R=200 \Omega$ by a current $I_{\mathrm{B}}$, and the voltage $V_{\mathrm{J}}$ across it is monitored to detect switching. Right: SEM micrographs of sample \#1 at different scales. In the upper-right panel, the brighter pads are gold electrodes, while the darker part constituting the loop is made out of aluminum. The whole structure is deposited on top of a polyimide layer on a metallic substrate. The gold electrodes form the shunting capacitor $C_{\mathrm{e}}$ through the metallic substrate, and also act as quasiparticle traps (see [40,41] for details). The junction (lower-left panel) is fabricated using the technique of double-angle evaporation through a suspended mask. This also leads on the suspended bridge side to an extraneous metallic bridge, as seen on the bottom-right panel.

\subsection{Measurement of the current-phase relation}

In the setup presented in Fig. 6 a Josephson junction acts as such a switch [39-41]. The atomic contact and the Josephson junction are embedded in a small superconducting loop, forming a device that was coined the "atomic-SQUID". This ancillary junction not only allows both biasing configurations but is also used to measure the loop current. The critical current of the Josephson junction is chosen to be much larger than the one of a typical aluminum one-atom contact $(\lesssim 50 \mathrm{nA})$ so that the SQUID essentially behaves like a slightly perturbed Josephson junction.

The $I(V)$ characteristic of the atomic contact, denoted $I_{\mathrm{AC}}(V)$, is obtained from that of the SQUID $I_{\mathrm{AS}}(V)$. In principle, in the region below the superconducting gap $|e V| \leqslant 2 \Delta$, the DC current flowing through the junction is expected to be zero. In practice however, a sizable current is observed experimentally in this region in the characteristic $I_{\mathrm{JJ}}(V)$ of the junction alone, which can be measured when the metallic bridge forming the atomic contact is fully open. Assuming that $I_{\mathrm{JJ}}(V)$ is not affected by the contact, $I_{\mathrm{AC}}(V)$ is then obtained by the subtraction:

$$
I_{\mathrm{AC}}(V)=I_{\mathrm{AS}}(V)-I_{\mathrm{JJ}}(V)
$$

which is then fitted using the MAR theory to obtain the transmissions.

The superconducting loop allows imposing a phase difference across the atomic contact by applying an external magnetic flux $\phi_{\text {ext }}$. If the loop is sufficiently small so that the screening flux can be neglected, ${ }^{5}$ the phase differences $\gamma$ (across the tunnel junction) and $\delta$ (across the atomic-size contact) are linked through:

$$
\delta=\gamma+2 \pi \frac{\phi_{\mathrm{ext}}}{\phi_{0}} \equiv \gamma+\varphi
$$

where $\varphi$ is the reduced flux threading the loop [42].

At zero temperature, the large Josephson junction would switch out of its zero-voltage state at a phase difference $\gamma=\frac{\pi}{2}$, when it carries exactly its critical current $I_{0}$. Assuming that the contributions of the junction and of the atomic contact can be separated, the critical current $I_{\mathrm{AS}}^{0}$ of the SQUID is the sum of the critical current $I_{0}$ of the junction and of the fluxdependent critical current $I_{\mathrm{AC}}(\delta)$ of the atomic contact:

$$
I_{\mathrm{AS}}^{0}(\varphi)=I_{0}+I_{\mathrm{AC}}\left(\frac{\pi}{2}+\varphi\right)
$$

Measuring the flux dependence of the critical current of the SQUID would thus be a direct way to probe the current-phase relation of the atomic contact. However, as mentioned before, in practice the critical current of a small Josephson device is not accessible. Due to fluctuations the system switches stochastically to a dissipative state before reaching this maximum current, and one simply measures the mean switching current of the SQUID.

The mean switching current is determined accurately from the switching probability $P$. This is measured using a train of bias current pulses of a given height and counting the number of pulses for which a voltage appears across the SQUID. We

\footnotetext{
5 The geometric inductance $L_{L}$ of the loop, typically $10 \mathrm{pH}$ is chosen to be negligible as compared to the inductance of both the Josephson junction $L_{J} \simeq 1 \mathrm{nH}$ and the atomic contact $L_{\mathrm{AC}} \simeq 10 \mathrm{nH}$. In this way, the phase-drop takes place essentially across these two last elements.
} 

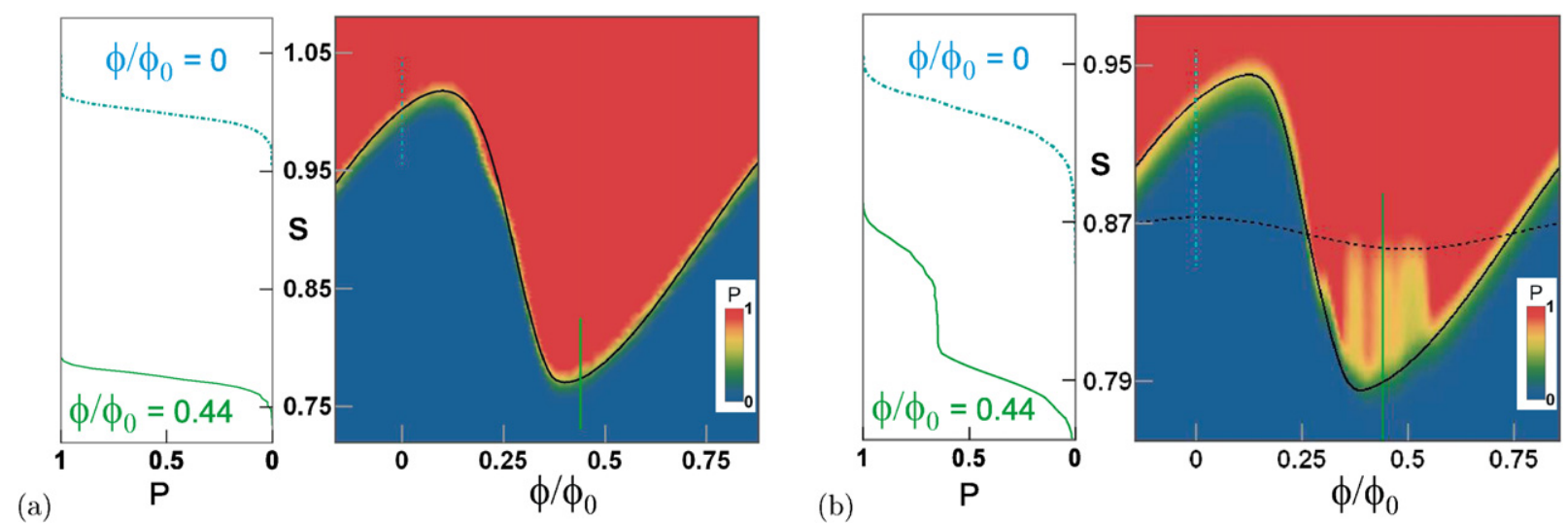

Fig. 7. (a) Left: Switching probability $P$ for an atomic SQUID obtained with sample \#1 as a function of the reduced bias current $s$, for two values of the external flux. Right: switching probability (color-coded) as a function of both the reduced bias current and flux. The two vertical lines correspond to the two curves plotted in the left panel. The full black line corresponds to the modulation curve $s^{*}(\varphi)$ calculated using the full PIN of the contact, taking into account the contribution of just the Andreev ground state in each channel. The PIN code of the atomic contact was $\{0.969,0.137\}$. (b) Left: Switching probability $P$ for an atomic-SQUID obtained with sample \#2 as a function of the reduced bias current $s$, for two values of the external flux. The upper curve is similar to those obtained in sample \#1 but the lower curve presents a clear intermediate plateau. The PIN code of the atomic contact was $\{0.994,0.097,0.096\}$. Right: switching probability (color-coded) as a function of both the reduced bias current and flux. The two vertical lines correspond to the two curves plotted in the left panel. The full line corresponds to the modulation curve $s^{*}(\varphi)$ calculated taking into account the contribution of the Andreev ground state of all the channels. The dashed line corresponds to the modulation curve calculated for $\{0.097,0.096\}$, which excludes the contribution of the most transmitted channel.

plot in the right panel of Fig. 7(a) the switching probability as a function of both the reduced bias current $s=I_{B} / I_{0}$ and the reduced flux $\varphi$ in the loop, for a particular atomic-contact obtained with sample \#1 (described in Fig. 6). The left panel of the same figure shows two examples of $P(s)$ for two different values of flux. One observes that the switching probability evolves very rapidly from 0 to 1 in a narrow range of bias current which depends on the applied flux. In the following, we denote $s^{*}$ the reduced bias current leading to a switching probability $P=0.5$, and refer to $s^{*}(\varphi)$ as the SQUID "modulation curve".

In order to calculate the expected modulation curve one considers the phase dynamics in the full Josephson potential of the SQUID in the presence of thermal fluctuations [39,43]. The potential is dominated by the Josephson energy of the junction but contains a contribution which consists of the sum of the ground sate Andreev energies of every channel of the atomic contact. As shown in the right panel of Fig. 7(a) this model works very well.

In conclusion, for all the contacts that we investigated using sample \#1 the current-phase relation measured at low temperatures is in quantitative agreement with the mesoscopic description of the Josephson effect, considering that only the ground Andreev state is occupied in each channel. The next step in order to achieve a comprehensive test of the theory would be to probe the excited Andreev states for example by directly performing spectroscopy of the transitions between Andreev levels. However, during our first attempts to do so, we observed unexpected but important phenomena which shed light on the microscopic nature of the system.

\subsection{Out-of-equilibrium effects}

The experiments presented in the previous sections were performed with atomic-SQUIDs obtained on sample \#1, which was fabricated on top of a metallic substrate and in which the on-chip lines connecting the SQUID to the outside world were made out of gold, a dissipative metal at low temperature. On the contrary in our first design to attempt microwave Andreev spectroscopy, in order to minimize dissipation which is expected to limit the lifetime of the excited Andreev levels [44] and thus broaden the spectral lines, we decided to fabricate the samples on Kapton substrates with all on-chip electrodes made out of aluminum. Apart from this change of material sample \#2 was almost identical to the previous one and in particular the critical current of the junction was $I_{0}=295 \mathrm{nA}$, less than $5 \%$ smaller than before. However, as we will discuss now, this change of material had strong consequences on the overall behavior of the system.

The right panel of Fig. 7(b) shows the measured switching probability as a function of both the flux and the reduced bias current for a SQUID with an atomic contact of transmissions $\{0.994,0.097,0.096\}$ in sample \#2. As compared to the previous sample (see Fig. 7(a)) the switching probability $P(s)$ does not vary sharply from 0 to 1 for all values of the flux. In some flux ranges, it displays a step as a function of the bias current, as shown for $\varphi=0.44$ in the left panel of the same figure. These features appear only in a region bounded by the expected modulation curve $s^{*}(\varphi)$ and by a second modulation curve calculated without the contribution of the highest transmitted channel. In other words, in this region the highest transmitted channel does not seem to always contribute to the total current through the contact. This is exactly what is expected if the two Andreev states of this channel are either both empty or both occupied, an occupation configuration achieved when a single quasiparticle is trapped in the Andreev doublet as discussed in Section 3.2. Since the Andreev states have energies below the superconducting gap the quasiparticles are easily trapped. The fact that this effect 


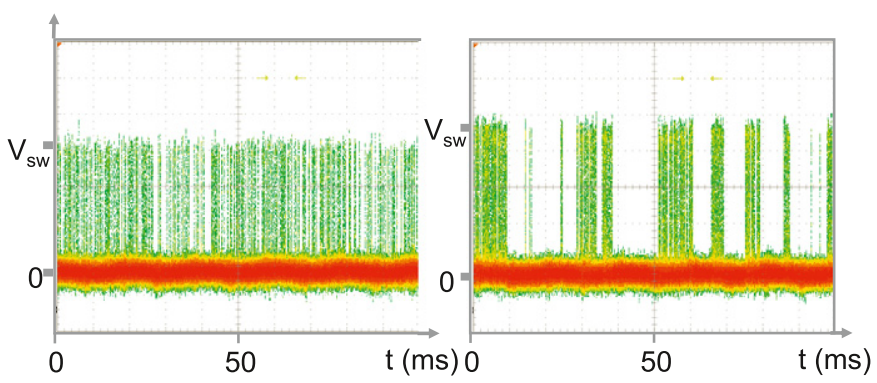

Fig. 8. Voltage across the atomic-SQUID as a function of time during a sequence of bias current pulses (50 $\mu$ s period) corresponding to a switching probability $P=0.2$. Each voltage pulse corresponds to a switching event. Left: at a flux value for which the $P(s)$ curve is normal. Right: Similar measurement, at a flux value for which $P(s)$ displays a step, showing intermittency attributed to the dynamics of single quasiparticle trapping and untrapping in the Andreev doublet.
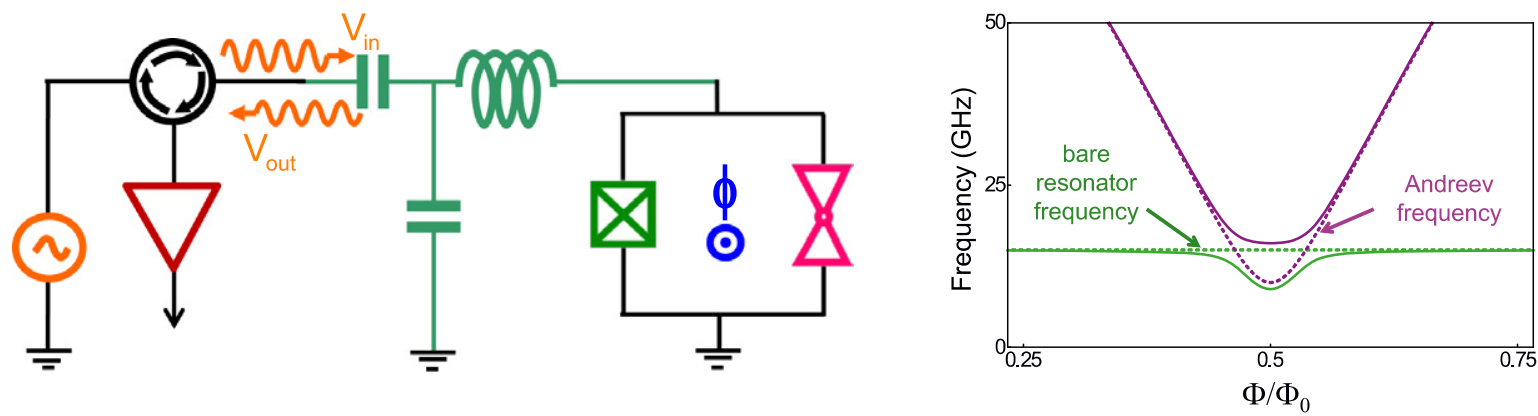

Fig. 9. Left: An atomic-SQUID coupled to a microwave resonator, represented here by the LC circuit (in green). The spectrum of the combined system is probed through microwave reflectometry by weakly coupling the resonator to the external setup through a small capacitor. The signal reflected by the sample is diverted by a circulator into a microwave amplifier. Right: Example of the expected spectrum (full lines) as a function of the magnetic flux threading the SQUID loop. The resonance frequency of the bare resonator (green dashed line) is chosen to be $15 \mathrm{GHz}$, the Andreev frequency (magenta dashed line) is for a channel of $\tau=0.995$, and the coupling energy is $h \times(5 \mathrm{GHz})$. Note the anti-crossing signaling the hybridization of the two quantum systems. (For interpretation of the references to color in this figure legend, the reader is referred to the web version of this article.)

only appears on samples having no large normal electrodes able to efficiently remove the quasiparticles strongly supports this hypothesis. Moreover, if trapping happens in a flux region for which the critical current gets enhanced by the double occupancy, subsequent current pulses of the same height would be unable to make the system switch. It is only after the quasiparticle gets untrapped that switching occurs again. This results in intermittency of switching as quasiparticles trap and untrap, as shown in Fig. 8. Recently, we have measured the full dynamics of trapping and found that the lifetime of trapped quasiparticles can be quite long, on the order of $100 \mu \mathrm{s}$ [45]. This phenomenon is reminiscent of the "poisoning" by a single quasiparticle observed in single Cooper-pair devices containing small superconducting islands in which the parity of the total number of electrons actually matters. However, in the case we discuss here quasiparticle trapping occurs in a constriction between two superconductors, a system containing no island at all and where charging energy does not play a role.

\section{Towards Andreev spectroscopy}

As shown in the previous section, up to now only the properties of the Andreev ground state have been probed in detail. Performing spectroscopy of the transition between two Andreev states is clearly the next important step which we are presently pursuing along two different lines.

\subsection{Microwave reflectometry}

The idea here is to couple an atomic SQUID to the electromagnetic field of a coplanar waveguide resonator (left panel of Fig. 9). By varying the flux threading the SQUID loop the Andreev transition frequency can be brought into resonance with one of the resonator's modes. This will result in the hybridization between the Andreev levels and the modes of the cavity (right panel of Fig. 9). The goal of the experiment would be to detect this hybridization, a strategy that has been implemented successfully for superconducting qubits [46]. The required technical developments are underway. 

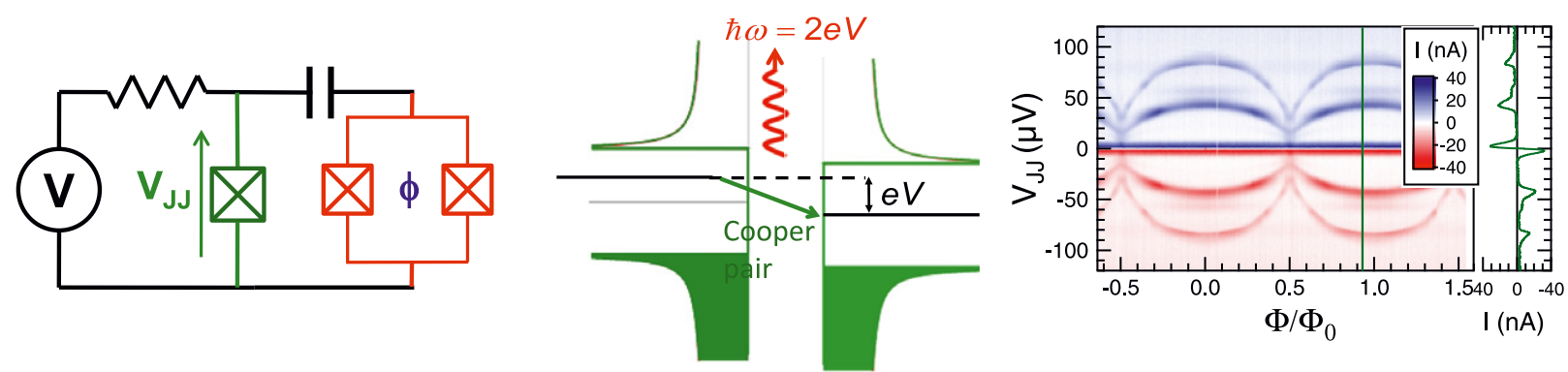

Fig. 10. Left: Experimental setup: a voltage biased Josephson junction capacitively coupled to a dc SQUID. Center: Density of states of the two superconducting electrodes of the junction, shifted by $\mathrm{eV}$. Cooper pairs can tunnel only if their energy drop $2 \mathrm{eV}$ is absorbed as photons in the modes of its electromagnetic environment. Right: Measured current (color-coded) through the junction as a function of the voltage $V_{\mathrm{JJ}}$ across it (vertical axis) and of the magnetic flux threading the SQUID (horizontal axis). The flux dependent lines correspond to the excitation of the plasma mode of the SQUID. An example of the junction $I V$ (for a flux $\phi \simeq .92 \phi_{0}$ ) is shown in the rightmost panel.

\subsection{Inelastic Cooper pair tunneling}

The second strategy that we are implementing to perform spectroscopy avoids these challenges by using an on-chip microwave source and detector. Usually there is no current through a Josephson junction voltage biased below twice the superconducting gap. However, current peaks can develop in its sub-gap $I(V)$ curve at voltages $2 e V_{i}=h v_{i}$ if the energy $2 e V$ lost by a Cooper pair tunneling across the barrier can be absorbed as photons by the modes $v_{i}$ of the electromagnetic environment of the junction, like for example in the case of Dynamical Coulomb Blockade [47,48]. By capacitively coupling an ancillary Josephson junction to the atomic-SQUID (see Fig. 10) we expect to detect in the $I-V$ of the junction the Andreev mode of the atomic contact. For the moment we have simply tested this idea on a conventional two-junction SQUID and detected, as shown in the right panel of Fig. 10, its plasma resonance corresponding to the oscillations of the LC circuit formed by the Josephson inductance of the junctions and their intrinsic capacitance.

\section{Conclusions}

Atomic-size contacts between metallic electrodes are now routinely obtained by a variety of techniques. Because the number of conduction channels they accommodate can be adjusted in-situ, and their corresponding transmission coefficients are amenable to precise measurement, these structures are model quantum point contacts which can be considered as a test-bed for mesoscopic physics.

Besides the experiments described here, we have also tested quantitatively the predictions for other transport phenomena: shot-noise in both the normal and superconducting states [49]; dynamical Coulomb blockade in the normal state [22,50]; the crossover between supercurrent and MAR dissipative current in voltage-biased superconducting contacts [51]; the response of superconducting contacts to microwave irradiation [52]. In all cases the agreement between theory and experiment is remarkable and the ensemble of results strongly supports the scattering theory of quantum transport.

In the superconducting state atomic contacts are the simplest possible weak-links and perfectly illustrate the key role played by the Andreev bound states in the Josephson effect. The Andreev states in a short single channel Josephson structure constitute a two-level system [53] that has been proposed as the basis for a new class of superconducting qubits [54-56,44]. What is particularly interesting and novel is that in contrast with all other superconducting qubits based on Josephson junction circuits [57] an Andreev qubit is a microscopic two level system more like spin qubits in semiconducting quantum dots. It can be viewed as a superconducting "quantum dot" possibly allowing manipulation of the spin degree of freedom of a single quasiparticle [37,58]. As reviewed in this work we have already characterized Andreev states by measuring the current they carry but the coherence properties of Andreev doublets are a key issue still to be addressed. The relaxation time of the excited state and the dephasing time of a quantum superposition of the two states have to be measured, understood and if possible controlled.

\section{Acknowledgements}

We gratefully acknowledge the expert technical support of Pief Orfila and Pascal Senat, the input and assistance of other members of the Quantronics group (Michel Devoret, Patrice Bertet and Denis Vion) and the crucial contributions of the graduate students and postdocs who participated in this research effort in the past: Elke Scheer, Ronald Cron, Peter vom Stein, Martin Chauvin, Maria Luisa Della Rocca, Benjamin Huard, Maciej Zgirski and Quentin Le Masne. We have enjoyed along the years fruitful collaborations with Nicolás Agraït, Juan Carlos Cuevas, Alfredo Levy Yeyati, Alvaro Martín Rodero, Gabino Rubio and Jan van Ruitenbeek, and greatly benefited from discussions with Joachim Ankerhold, Sebastián Bergeret, Hermann Grabert, John Martinis and Vitaly Shumeiko. 


\section{References}

[1] R.A. Webb, S. Washburn, C.P. Umbach, R.B. Laibowitz, Observation of he Aharonov-Bohm oscillations in normal-metal rings, Physical Review Letters 54 (1985) 2696.

[2] R. Landauer, Electrical resistance of disordered one-dimensional lattices, Philosophical Magazine 21 (1970) $863-867$.

[3] T. Martin, R. Landauer, Wave-packet approach to noise in multichannel mesoscopic systems, Physical Review B 45 (1992) 1742.

[4] Y. Nazarov, Y.M. Blanter, Quantum Transport: Introduction to Nanoscience, Cambridge University Press, Cambridge, UK, New York, 2009.

[5] B.J. van Wees, H. van Houten, C.W.J. Beenakker, J.G. Williamson, L.P. Kouwenhoven, D. van der Marel, C.T. Foxon, Quantized conductance of point contacts in a two-dimensional electron gas, Physical Review Letters 60 (1988) 848.

[6] D.A. Wharam, T.J. Thornton, R. Newbury, M. Pepper, H. Ahmed, J.E.F. Frost, D.G. Hasko, D.C. Peacock, D.A. Ritchie, G.A.C. Jones, One-dimensional transport and the quantisation of the ballistic resistance, Journal of Physics C: Solid State Physics 21 (1988) L209-L214.

[7] N. Agraït, A. Levy Yeyati, J.M. van Ruitenbeek, Quantum properties of atomic-sized conductors, Physics Reports 377 (2003) 81.

[8] D.M. Eigler, C.P. Lutz, W.E. Rudge, An atomic switch realized with the scanning tunnelling microscope, Nature 352 (1991) $600-603$.

[9] J. Moreland, J.W. Ekin, Electron tunneling experiments using Nb-Sn "break" junctions, Journal of Applied Physics 58 (1985) 3888.

[10] C.J. Muller, J.M. van Ruitenbeek, L.J. de Jongh, Experimental observation of the transition from weak link to tunnel junction, Physica C: Superconductivity 191 (1992) 485-504.

[11] E. Scheer, P. Joyez, D. Esteve, C. Urbina, M.H. Devoret, Conduction channel transmissions of atomic-size aluminum contacts, Physical Review Letters 78 (1997) 3535.

[12] J.M. van Ruitenbeek, A. Alvarez, I. Piñeyro, C. Grahmann, P. Joyez, M.H. Devoret, D. Esteve, C. Urbina, Adjustable nanofabricated atomic size contacts, Review of Scientific Instruments 67 (1996) 108.

[13] G. Rubio, N. Agraït, S. Vieira, Atomic-sized metallic contacts: Mechanical properties and electronic transport, Physical Review Letters 76 (1996) 2302.

[14] G. Rubio-Bollinger, P. Joyez, N. Agraït, Metallic adhesion in atomic-size junctions, Physical Review Letters 93 (2004) 116803.

[15] T.N. Todorov, A.P. Sutton, Jumps in electronic conductance due to mechanical instabilities, Physical Review Letters 70 (1993) 2138.

[16] M. Brandbyge, J. Schiotz, M.R. Sorensen, P. Stoltze, K.W. Jacobsen, J.K. Norskov, L. Olesen, E. Laegsgaard, I. Stensgaard, F. Besenbacher, Quantized conductance in atom-sized wires between two metals, Physical Review B 52 (1995) 8499.

[17] M.R. Sorensen, M. Brandbyge, K.W. Jacobsen, Mechanical deformation of atomic-scale metallic contacts: Structure and mechanisms, Physical Review B 57 (1998) 3283.

[18] A.I. Yanson, G.R. Bollinger, H.E. van den Brom, N. Agraït, J.M. van Ruitenbeek, Formation and manipulation of a metallic wire of single gold atoms, Nature 395 (1998) 783-785.

[19] H. Ohnishi, Y. Kondo, K. Takayanagi, Quantized conductance through individual rows of suspended gold atoms, Nature 395 (1998) $780-783$.

[20] V. Rodrigues, D. Ugarte, Real-time imaging of atomistic process in one-atom-thick metal junctions, Physical Review B 63 (2001) 073405.

[21] H. Masuda, K. Monna, T. Matsuda, T. Kizuka, e-Journal of Surface Science and Nanotechnology 7 (2009) 549-552.

[22] R. Cron, Atomic contacts: A test-bed for mesoscopic physics, PhD thesis, Université Pierre et Marie Curie, Paris, France, 2001. Available at http://tel. archives-ouvertes.fr/tel-00001329/fr/.

[23] T.M. Klapwijk, G.E. Blonder, M. Tinkham, Explanation of subharmonic energy gap structure in superconducting contacts, Physica B+C 109-110 (1982) 1657-1664.

[24] G.E. Blonder, M. Tinkham, T.M. Klapwijk, Transition from metallic to tunneling regimes in superconducting microconstrictions: Excess current, charge imbalance, and supercurrent conversion, Physical Review B 25 (1982) 4515.

[25] G.B. Arnold, Superconducting tunneling without the tunneling hamiltonian. II. Subgap harmonic structure, Journal of Low Temperature Physics 68 (1987) 1 .

[26] D. Averin, A. Bardas, Ac Josephson effect in a single quantum channel, Physical Review Letters 75 (1995) 1831.

[27] J.C. Cuevas, A. Martín-Rodero, A.L. Yeyati, Hamiltonian approach to the transport properties of superconducting quantum point contacts, Physical Review B 54 (1996) 7366 .

[28] J.J. Riquelme, L.d.l. Vega, A.L. Yeyati, N. Agraït, A. Martin-Rodero, G. Rubio-Bollinger, Distribution of conduction channels in nanoscale contacts: Evolution towards the diffusive limit, Europhysics Letters (EPL) 70 (2005) 663-669.

[29] E. Scheer, N. Agraït, J.C. Cuevas, A. Levy Yeyati, B. Ludoph, A. Martín-Rodero, G. Rubio Bollinger, J.M. van Ruitenbeek, C. Urbina, The signature of chemical valence in the electrical conduction through a single-atom contact, Nature (London) 394 (1998) 154.

[30] E. Scheer, W. Belzig, Y. Naveh, M.H. Devoret, D. Esteve, C. Urbina, Proximity effect and multiple Andreev reflections in gold atomic contacts, Physical Review Letters 86 (2001) 284.

[31] B.D. Josephson, Possible new effects in superconductive tunnelling, Phys. Lett. 1 (1962) 251.

[32] A.A. Golubov, M.Y. Kupriyanov, E. Il'ichev, The current-phase relation in Josephson junctions, Reviews of Modern Physics 76 (2004) 411.

[33] A. Furusaki, M. Tsukada, A unified theory of clean Josephson junctions, Physica B: Condensed Matter 165-166 (1990) 967-968.

[34] C.W.J. Beenakker, H. van Houten, Josephson current through a superconducting quantum point contact shorter than the coherence length, Physical Review Letters 66 (1991) 3056.

[35] A.F. Andreev, Sov. Phys. JETP 19 (1964) 1228.

[36] P.F. Bagwell, Suppression of the Josephson current through a narrow, mesoscopic, semiconductor channel by a single impurity, Phys. Rev. B 46 (1992) $12573-12586$

[37] N.M. Chtchelkatchev, Y.V. Nazarov, Andreev quantum dots for spin manipulation, Physical Review Letters 90 (2003) 226806.

[38] M.F. Goffman, R. Cron, A. Levy Yeyati, P. Joyez, M.H. Devoret, D. Esteve, C. Urbina, Supercurrent in atomic point contacts and Andreev states, Physical Review Letters 85 (2000) 170.

[39] M.L. Della Rocca, M. Chauvin, B. Huard, H. Pothier, D. Esteve, C. Urbina, Measurement of the current-phase relation of superconducting atomic contacts, Physical Review Letters 99 (2007) 127005-4.

[40] B. Huard, Interactions between electrons, mesoscopic Josephson effect and asymmetric current fluctuations, PhD thesis, Université Pierre et Marie Curie, Paris, France, 2006. Available at http://tel.archives-ouvertes.fr/tel-00119371/fr/.

[41] M. Chauvin, Effet Josephson dans les contacts atomiques (The Josephson effect in atomic contacts), PhD thesis, Université Pierre et Marie Curie, Paris, France, 2005. Available at http://tel.archives-ouvertes.fr/tel-00107465/fr/.

[42] V. Lefevre-Seguin, E. Turlot, C. Urbina, D. Esteve, M.H. Devoret, Thermal activation of a hysteretic dc superconducting quantum interference device from its different zero-voltage states, Physical Review B (Condensed Matter and Materials Physics) 46 (1992) 5507-5522.

[43] Q. Le Masne, Asymmetric current fluctuations and Andreev states probed with a Josephson junction, PhD thesis, Université Pierre et Marie Curie, Paris, France, 2009. Available at http://tel.archives-ouvertes.fr/tel-00482483/fr/.

[44] M.A. Despósito, A. Levy Yeyati, Controlled dephasing of Andreev states in superconducting quantum point contacts, Physical Review B 64 (2001) 140511.

[45] M. Zgirski, L. Bretheau, Q. Le Masne, H. Pothier, D. Esteve, C. Urbina, Evidence for long-lived quasiparticles trapped in superconducting point contacts, Physical Review Letters 106 (2011) 257003. 
[46] A. Blais, R. Huang, A. Wallraff, S.M. Girvin, R.J. Schoelkopf, Cavity quantum electrodynamics for superconducting electrical circuits: An architecture for quantum computation, Physical Review A 69 (2004) 62320

[47] T. Holst, D. Esteve, C. Urbina, M.H. Devoret, Effect of a transmission line resonator on a small capacitance tunnel junction, Physical Review Letters 73 (1994) 3455-3458.

[48] M. Hofheinz, F. Portier, Q. Baudouin, P. Joyez, D. Vion, P. Bertet, P. Roche, D. Esteve, Bright side of the Coulomb blockade, Physical Review Letters 106 (2011) 217005.

[49] R. Cron, M.F. Goffman, D. Esteve, C. Urbina, Multiple-charge-quanta shot noise in superconducting atomic contacts, Physical Review Letters 86 (2001) $4104-4107$.

[50] R. Cron, E. Vecino, M.H. Devoret, D. Esteve, P. Joyez, A. Levy Yeyati, A. Martin-Rodero, C. Urbina, Dynamical Coulomb blockade in quantum point contacts, in: Electronic Correlations: From Meso- to Nano-Physics, EDP Sciences, 2001.

[51] M. Chauvin, P. vom Stein, D. Esteve, C. Urbina, J.C. Cuevas, A.L. Yeyati, Crossover from Josephson to multiple Andreev reflection currents in atomic contacts, Physical Review Letters 99 (2007) 067008.

[52] M. Chauvin, P.v. Stein, H. Pothier, P. Joyez, M.E. Huber, D. Esteve, C. Urbina, Superconducting atomic contacts under microwave irradiation, Physical Review Letters 97 (2006) 067006.

[53] D.A. Ivanov, M.V. Feigel'man, Two-level hamiltonian of a superconducting quantum point contact, Phys. Rev. B 59 (1999) $8444-8446$.

[54] A. Zazunov, V.S. Shumeiko, E.N. Bratus', J. Lantz, G. Wendin, Andreev level qubit, Physical Review Letters 90 (2003) 087003.

[55] A. Zazunov, V.S. Shumeiko, G. Wendin, E.N. Bratus', Dynamics and phonon-induced decoherence of Andreev level qubit, Physical Review B (Condensed Matter and Materials Physics) 71 (2005) 214505.

[56] J. Lantz, V.S. Shumeiko, E. Bratus, G. Wendin, Flux qubit with a quantum point contact, Physica C: Superconductivity 368 (2002) 315.

[57] G. Wendin, V.S. Shumeiko, Quantum bits with Josephson junctions (Review article), Low Temperature Physics 33 (2007) 724.

[58] J. Michelsen, V.S. Shumeiko, G. Wendin, Manipulation with Andreev states in spin active mesoscopic Josephson junctions, Physical Review B 77 (2008) 184506. 